\title{
Using the Hybrid Simulation for Early User Evaluations of Pervasive Interactions
}

\author{
Karin Leichtenstern, Elisabeth André \\ Augsburg University, Universitätsstr. 6a, \\ 86159 Augsburg \\ \{leichtenstern, \\ andre\}@informatik.uni-augsburg.de
}

\author{
Matthias Rehm \\ Aalborg University, Niels Jernes Vej 14, \\ 9000 Aalborg \\ \{matthias\}@imi.aau.dk
}

\begin{abstract}
To reach a good user-friendliness, knowledge about user requirements is crucial in the development process of a product. The sooner the knowledge is achieved via user evaluations, the more money and time can be saved. In this paper we investigate an approach called hybrid simulation for the early stages evaluation of mobile applications where real mobile phones are used as interaction devices to a virtualised simulation of a pervasive environment. On the first sight, the method is cheap, easy and quick to use as well as more realistic compared to a virtual simulation only approach. In order to receive a more detailed insight in potential benefits and problems of the method, we performed a user study and compared results of a traditional laboratory study with the results of a study performed with the hybrid simulation.
\end{abstract}

\section{ACM Classification Keywords}

H.5.2 Information Interfaces and Presentation: User Interfaces-Evaluation/Methodology, Prototyping, UserCentred Design, Input Devices and Strategies

\section{Author Keywords}

User-centred Design, Early Stages Evaluation Method, Hybrid Simulation, Pervasive Interface, Mobile Phones

\section{INTRODUCTION}

The idea of Ubiquitous Computing and its synonym Pervasive Computing [36, 8] is to make the computer invisible in our everyday life in order to enable interactions with everything, everywhere at anytime [7]. The users can either directly interact with physical objects in their environment (e.g. interactive surfaces) or make use of interaction devices as medium to the objects. By this mean the user can request services for the object, such as the current status of a home appliance. The

This is the author's version of the work. It is posted here for your personal use. Not for redistribution. The definitive Version of Record was published in:

NordiCHI 2010, October 16-20, 2010, Reykjavik, Iceland.

Copyright 2010 ACM ISBN: 978-1-60558-934-3 first real interaction devices to physical objects are mobile phones. Almost everybody owns a mobile phone and takes it around constantly. Recent phones support novel hardware and network facilities that enable different interactions to pervasive environments, such as an interaction with a public display to download displayed information. Ballagas and colleagues [1] give a comprehensive overview about the different input channels available with todays smart phones (e.g. the phone's built-in camera or accelerometer) that can be used for mobile interaction techniques. Despite the promising potential of using mobile phones as interaction devices to pervasive environments, some problems emerge that need to be solved.

Compared to the development for desktop settings, the development of mobile applications in the context of Pervasive Computing adds new layers of complexity. Ensuring user-friendliness in this context is a challenge due to a number of reasons. For instance, the developer has to cope with limited input and output capabilities of the mobile devices [8]. Moreover, the contextual setting of the interaction is unpredictable due to the mobility of the user [11]. In terms of Dey [5], information about the user's situation is often unknown which includes knowledge about the requirements of users as well as their typical behaviour and preferences in the corresponding situations. To tackle these challenges, the application of the user-centred development process $[33,25,16]$ is a possibility to obtain a good design for mobile applications.

A characteristic feature of this process is an iterative prototyping that includes several iterations of designing and implementing prototypes along with continuous executions and analyses of user evaluations with the generated prototypes. In terms of interface design, a prototype represents a partial simulation of a product with respect to its final appearance and behaviour [13]. The execution of user evaluations with the prototypes can provide interface developers with the relevant knowledge about the users and their requirements as well as behaviour. Traditionally, these user evaluations are either performed in-situ (field study) or simulated in a laboratory. The field studies are often postponed to the end of the development process because they typically require fully implemented applications. Addition- 
ally, they are often expensive and difficult to organise and conduct. Compared to field tests, laboratory studies are easier to organize and conduct but often less realistic because it is difficult to ensure a test setting that is as similar as possible to the field setting. Additionally, it is often difficult to recruit a large number of subjects for traditional laboratory studies since laboratory studies normally require the physical presence of the subjects.

In this paper, we describe and investigate the concept of using the hybrid simulation as an evaluation method in early stages of the user-centred development of mobile applications. After a literature review of current evaluation methods, the concept and implementation of the hybrid simulation is described more detailed. Finally, a comparative user study is presented in order to reveal potential benefits but also problems of the hybrid simulation as evaluation method at early stages of the user-centred development process.

\section{FIELD AND LABORATORY STUDIES}

The most reasonable evaluation method is the execution of a field test in the real environment of the user. Different field studies with mobile applications have been executed and described [11, 29, 30]. For example, Häkkila and colleagues [10] performed a user study in the city centre of Oulu. They used different applications, such as an event calendar or guidance service during their field test. This test was performed as a diary study combined with user interviews which gave valuable data of the user's behaviour.

There is evidence that field tests provide very realistic and valuable data because they are performed in the real world with real contextual constraints. The execution of field tests, however, also causes problems. Field studies might lead to uncontrolled contextual settings rendering the outcome useless. Moreover, field tests are often time-consuming to coordinate and conduct as well as quite expensive. Thus, the idea is to simulate parts of the real setting in a more controlled environment and conduct laboratory studies instead. A review [17] showed that $71 \%$ of the user studies for mobile phones are conducted as laboratory studies.

But do laboratory studies really substitute field studies? Several comparisons of field and laboratory studies have aimed at answering this question [15, 6, 19, 18, 12]. For instance, Kjeldskov and Stage [19, 18] searched for appropriate techniques which enable evaluations of mobile applications in the field and in the laboratory. They found some differences between field and laboratory studies, such as the social comfort of use but pointed out most basic usability problems as similar. Other studies (e.g. Kaikkonen and colleagues [15]) validated these results. They revealed laboratory studies as sufficient in most cases due to the fact that field studies do not often provide an added value.
Overall, the results showed that field tests cannot be completely substituted by laboratory studies and should be used at least at the end of the development process to investigate specific user behaviour in different contextual settings. Laboratory studies, however, can be used during minor iterations of the design process if appropriate evaluation methods and techniques are applied. But at that point a problem emerges. Duh and colleagues [6, 28] as well as Kjeldskov [19, 18] see a lack of appropriate evaluation methods and techniques in laboratory settings. In particular when investigating user behaviour in early stages of the development process, there is a need for appropriate methods [6]. Sá and Carricio reflected different low-fidelity evaluation techniques for user studies and discuss their feasibility $[4,3]$. They also see a lack of appropriate techniques in early stages of the design process that reduce the costs, required time and organisational effort.

\section{VIRTUAL AND HYBRID SIMULATIONS}

One idea is to apply virtual simulations at the beginning of the user-centred development process. Simulations via virtual worlds can improve the development process [20] because they can mediate ideas of new products and support first user evaluations. In the context of Pervasive Computing, a literature review showed a tendency to apply virtual simulations in order to investigate the pervasive environment itself and its performance [35, 32]. The use of virtual simulations for the investigation of pervasive interaction devices has not been focused so far. The projects that do address this aspect $[14,24$, $35,2]$, mostly used virtual simulations which directly included the use of the interaction device into the virtual world. Mobile devices are not longer physical available for interactions anymore. Instead, they are just virtually represented and have to be controlled via keyboard or mouse which leads to a disruption of the real usage of the device. For example, Manninen [24] used virtual representations of interaction devices in his setting. His main objective was to easily develop and test different virtual worlds and their input devices. Barton [2] is also interested in similar objectives. He developed UbiWise that is a simulation tool for Ubiquitous Computing applications. This simulation tool helps to investigate applications that use cameras or mobile phones as interaction devices. As the devices are only represented in a $3 \mathrm{D}$ virtual world, the user has to interact via the traditional input methods (e.g. mouse) which mean a marginal mapping to the real usage of the device and the application. Certainly, there is a need to consider the level of immersion that can often not be met by a simulation alone.

To solve the problem of the insufficient mapping, we searched for other approaches of simulations which involve at least parts of the real world. Morla and Davies [26] present such work. They used simulations to test a location-based application for health monitoring. Using their simulation environment, they can virtually evaluate the performance of sensors attached to a wearable 
medical monitoring system. Although their main objective was not to investigate user interactions with pervasive environments but instead the performance of the pervasive environment, Morla and Davies' work gives a first impression of a so-called hybrid simulation. Hybrid simulation means an integration and combination of the real and the virtual world. The medical monitoring system is not simulated and really reacts on the artificially generated contexts. A similar approach called dual reality is introduced by Lifton and colleagues [23]. They used Second Life as a virtual visualisation tool of streams which are generated from real world sensors (e.g. temperature sensors). Driving simulators ${ }^{1}$ also aim at the idea of the hybrid simulation. The users interact with a real steering wheel and dashboard while they are driving through a virtually presented test route. In our work, we do not use the virtual world as a visualisation platform for the performance of real devices as Davies and Lifton. We apply the virtual world as an evaluation platform for user studies similarly as used for driving simulators but we use real mobile phones as interaction devices and a virtual simulation of the pervasive environment. A similar idea is also described by Haesen et al. [9]. They used a virtual simulation of a museum to execute a user study where real mobile phones are applied to interact with the virtualised museum. Haesen et al. consider the concept of the hybrid simulation as a promising new evaluation technique in early stages of the user-centred design process. In contrast to them, we use a well-known platform for the virtual simulation of a pervasive environment (Second Life) as well as a tool to quickly and easily generate prototypes (MoPeDT). Additionally, we executed a user study to get insights to potential benefits but also problems when applying the hybrid simulation.

\section{HYBRID SIMULATION}

Applying the concept of the hybrid simulation to our domain, the user still interacts with the pervasive environment via a real mobile phone but the pervasive environment is now virtually represented in a simulation.

\section{Pervasive Interfaces Developement}

The pervasive interface development requires combined knowledge of software and usability engineering. This includes complex aspects, such as the implementation of the network communication and the interface programming of the mobile phone. As a consequence, the implementation of the different prototypes often takes too much time. There is a need to reduce the implementation time in order to be able to spend more time on the evaluation of the prototypes. To reduce the implementation time and to improve the interface's friendliness, the literature approves the need for software tools, e.g $[27]$.

We developed a tool called MoPeDT (Pervasive Development Toolkit for Mobile Phones) [22] that supports

\footnotetext{
${ }^{1}$ http://www.carrsq.qut.edu.au/simulator/
}

user interface developers in the user-centred prototyping of pervasive interfaces for mobile phones. Applying MoPeDT, applications for mobile phones can be designed, evaluated and analysed that support different pervasive interaction techniques [21] for the interaction with physical objects (e.g. products in a shopping store, objects of art in a museum or home appliances). For instance, the mobile phone and its built-in $\mathrm{NFC}^{2}$ reader can be applied to select a home appliance via an RFID tag that is attached to it. Further supported interaction techniques of MoPeDT utilize the user's speech or location for interactions with physical objects. After having selected a physical object, different services and their contents are loaded from the database and displayed on the mobile phone, such as a detailed description of the selected object or information about the object's origin. The idea to interact with physical objects and provide services to these objects follows the idea of Pervasive Computing.

In order to support interface developers to user-centred develop and evaluate prototypes in the term of Pervasive Computing, MoPeDT employs a client-server architecture and software modules [21, 22]. For instance, a software module for mobile phones is used to generate prototypes of a mobile application. Additionally, whenever tool-supported user studies have to be conducted, the architecture's component called evaluator is applied.

Other components of the architecture are the main server, the database as well as the sensors and actuators. The database persistently stores all information about the physical objects (e.g. a detailed description) that can be requested by the mobile user via the main server. The sensor and actuator can be used to collect or display additional information about the pervasive environment. Sensors, such as a temperature or a humidity sensor can collect, interpret and broadcast contexts to the main server. The main server can forward these contexts to interested users or actuators. Actuators, such as a public display can receive and display contexts or other information (e.g. video content).

Overall, using MoPeDT, different prototypes of pervasive interfaces for mobile phones can be implemented and user tests can be performed in real world settings of a pervasive environment as well as in a laboratory setting which has now been extended to also support user evaluations in hybrid simulations.

\section{Hybrid Simulation of Pervasive Interfaces}

MoPeDT can also be used to generate prototypes for mobile phones which should be evaluated via the hybrid simulation. Therefore, some adaptations are required. Figure 1 illustrates the architecture of MoPeDT which is required to perform the hybrid simulation. The main difference to the general architecture of MoPeDT [21, 22 ] is the shift of the pervasive environment from the

\footnotetext{
${ }^{2}$ http://www.nfc-forum.org/
} 


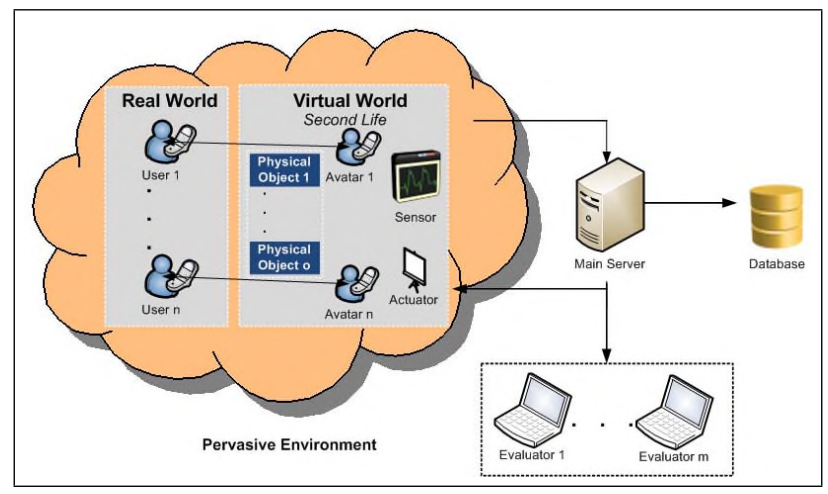

Figure 1. MoPeDT's extended architecture that illustrates the Shift of the Pervasive Environment in the Virtual World.

real world to the virtual world using a platform for a virtual world simulation, such as Second Life. Now, this simulation contains the virtual representation of all physical objects. All information about the physical objects is still stored in the database and can be accessed by the users with their real mobile phones. Thus, the user still makes use of a real mobile phone as an interface to the pervasive computing environment even though the physical objects are not longer physically present. Another difference to the former setting is the need for a representation of the user in the virtual world. With this avatar the user can interact via the keyboard within the virtualised pervasive environment, such as moving around to get closer to physical objects. These interactions can also create contextual information like a location event which is sent to the main server and then forwarded to interested mobile devices. The next section gives more information about these aspects and the simulated pervasive environment.

\section{Simulation of the Pervasive Environment}

To simulate the pervasive environment, we make use of an open source version of Second Life, which is called Open Simulator ${ }^{3}$. Open Simulator allows setting up one's own virtual world that behaves exactly like Second Life and can be accessed with the same viewers. Thus, in the remainder of this paper we will use Second Life and Open Simulator as synonyms.

Second Life represents one of the first massive 3D multiplayer platforms which is not primarily concerned with gaming but aims at establishing a general virtual meeting place. Thus, every conceivable type of interaction is in principle possible, be it buying or selling virtual or real goods, be it playing out as a real DJ in a virtual club, or be it pervasive interactions in an Ambient Intelligence landscape. Central feature of Second Life is the use of avatars which represent the real user in the virtual environment. We [31] have shown that Second Life can serve as an evaluation platform for multi agent systems involving the user in her natural environ-

\footnotetext{
${ }^{3}$ http://opensimulator.org
}

ment. We propose to employ Second Life to simulate a real environment which has been augmented for context dependent interactions. Apart from setting up the simulation server, three steps are necessary for simulating a pervasive environment in a hybrid simulation. The environment itself has to be modelled, it has to be equipped with physical objects and sensors, and it has to allow for communicating with the outside world such as the real mobile device.

\section{Modelling the Environment}

The most basic requirement is a virtual representation of the environment, in which the user evaluation should take place. To this end, standard modelling tools can be employed making it necessary to import the resulting models in Second Life or in-world modelling tools are used that supply basic functionalities. Figure 4 provides knowledge about the environment which was created for our study. Based on the requirements from our previous user evaluation which constitutes our benchmark (see Section The Reference User Study), a living room and a bathroom were modelled along with the furniture and devices like DVD player and TV.

\section{Making the Environment Pervasive}

The challenge of a hybrid simulation is to realise the complex interplay between sensors, physical objects, and the mobile device, which can be seen as the inherent characteristic of a pervasive environment. The general idea is to use the real mobile for the interaction with the virtual world. This is not always possible.

In our proof of concept study, objects are equipped with RFID tags to allow NFC with the mobile phone. Creating a virtual RFID tag is no challenge but of course this tag cannot be read out by the real mobile device. Thus, it is necessary to create a virtual representation of the mobile device for some of the contextual input. In the current version of our hybrid simulation, a virtual mobile device is used for registering the contextual input that is provided by the simulated environment. The real mobile device handles the output and the user interactions. Details are given in the next section.

Apart from the virtual representation of the mobile device, we have realised physical objects which are triggered by user actions, sensors which are triggered by changes in the environment, and additional contextual input. To create physical objects, home appliances (e.g. TV and DVD player) were augmented with RFID tags allowing for near field communication (NFC), and with virtual IR sensors to register remote activation (see Section The Reference User Study for information on the interaction techniques). A virtual temperature sensor was necessary to register the effects of manipulating the heater. To this end, a model of the heater and its radiation was realised. To allow for indoor localisation of the user, WLAN access points have been installed in the environment. By measuring the signal strength, the user's location can be approximated. Again, a model for the 
radiation was integrated. In both cases, a simple linear degression was chosen as a suitable model (see Figure 2).

\section{Communication}

Two types of communications take place in our pervasive environment. Sensors directly communicate with the main server of the MoPeDT architecture. Physical objects on the other hand communicate with the virtual representation of the mobile phone, which in turn communicates with the main server (see Figure 1). So far, communication is based on the in-world scripting language. In order to allow for a stronger inclusion of the real mobile phone also for registering the virtual input that is provided by the simulated environment, it seems inevitable to integrate a context class in the Open Simulator server.

The only sensor in our reference study which registers environmental changes is the temperature sensor. Thus, in each room, one sensor is placed that registers the local temperature according to the settings of the heater and its degression model (see Figure 2). Each sensor sends this information along with its ID as an HTTP request to the main server.

The home appliances have been augmented with RFID tags and IR sensors. Having identified a specific physical object activates its context on the mobile device, i.e. the services of this object become available to the user. To read out an RFID tag, the user moves her avatar towards the physical object. The avatar is holding the virtual representation of the mobile phone. The phone serves as a virtual RFID reader, simulating NFC. Thus, if the RFID tag is in a certain range (less than $30 \mathrm{~cm}$ ) of the mobile, its ID is registered by the mobile device which sends a HTTP request to the main server containing its own ID and the object's ID. The IR sensor allows remote activation of an object's context. To achieve this goal, the user has to point the virtual mobile in the direction of the physical object (see Figure 4). Having been activated, the sensor sends the object's ID via virtual Bluetooth to the mobile which in turn sends it to the main server via an HTTP request.

The environment might also provide additional information that has to be actively transformed into contextual input. The indoor localisation in our scenario is of this type. WLAN access points have been installed in the environment (see Figure 2), which are utilized to calculate the rough location of the user. This location is the room the user is currently in. Thus, the room sets the interaction context by making only the services available on the real mobile phone which is defined by the appliances in this room. To estimate the user's location, the virtual representation of the mobile phone registers the signal strength of the WLAN access points at the user's current location and triangulates this location. The mobile's ID is sent along with the location ID to the main server.
THE HYBRID SIMULATION OF A SMART ENVIRONMENT

Morla and Davies [26] describe several requirements of a hybrid simulation (e.g. the application of the device's intrinsic code) which we considered as fulfilled when combining the features of MoPeDT with Second Life. Using MoPeDT we can upload the intrinsic code of the mobile phone application to physical devices which enables live user interactions on real mobile phones. At the same time, contexts can be generated via the user and his mobile phone as well as via the connected real or simulated sensors. All user evaluations can be logged and reproduced via MoPeDT's architecture and the supported evaluation component. Even so, to get more insights in benefits and problems of using the hybrid simulation for early user studies, we decided to compare the results of a user study performed in a traditional laboratory setting with the results achieved by using the hybrid simulation. To this end, we implemented the scenario of the reference study as a hybrid simulation.

\section{The Reference User Study}

We conducted the reference study as a traditional real world simulation of a smart environment in a laboratory [34]. The main objective of this study was finding out whether users apply different mobile interaction techniques dependent on contextual conditions in a smart environment. In our setting, the smart environment contained several physical objects (e.g. a TV or a heater) which could be addressed and controlled via a mobile phone. For example, the mobile phone could be applied as a remote control to change the status of the heater by switching it on or off or by changing its temperature. In the context of the reference study, we investigated the use of the mobile interaction techniques: touching, pointing and scanning. When using the techniques touching or pointing the user has to physically touch or point at the intended physical object with the mobile phone in order to address it. Scanning is a technique to address a physical object by using the mobile phone's graphical user interface and select the intended physical object out of the detected and graphically listed physical objects.

The reference study was performed with 20 people in a living room of a smart environment. All participants were sitting on a couch while they had to solve four different tasks in order to call a service of the intended physical object under different context conditions. (1) First, the user had line of sight to the physical object. The distance to the physical object was about three meters. (2) For the second task, the users were in front of the physical object. The distance to the physical object was about ten centimetres. (3) For the third task, the user did not have line of sight to the physical object. The physical object was located in another room and the distance was about 20 meters. (4) Finally, the user did not have a line of sight to the physical object. The physical object was located in the same room. The distance to the physical object was about four meters. To 


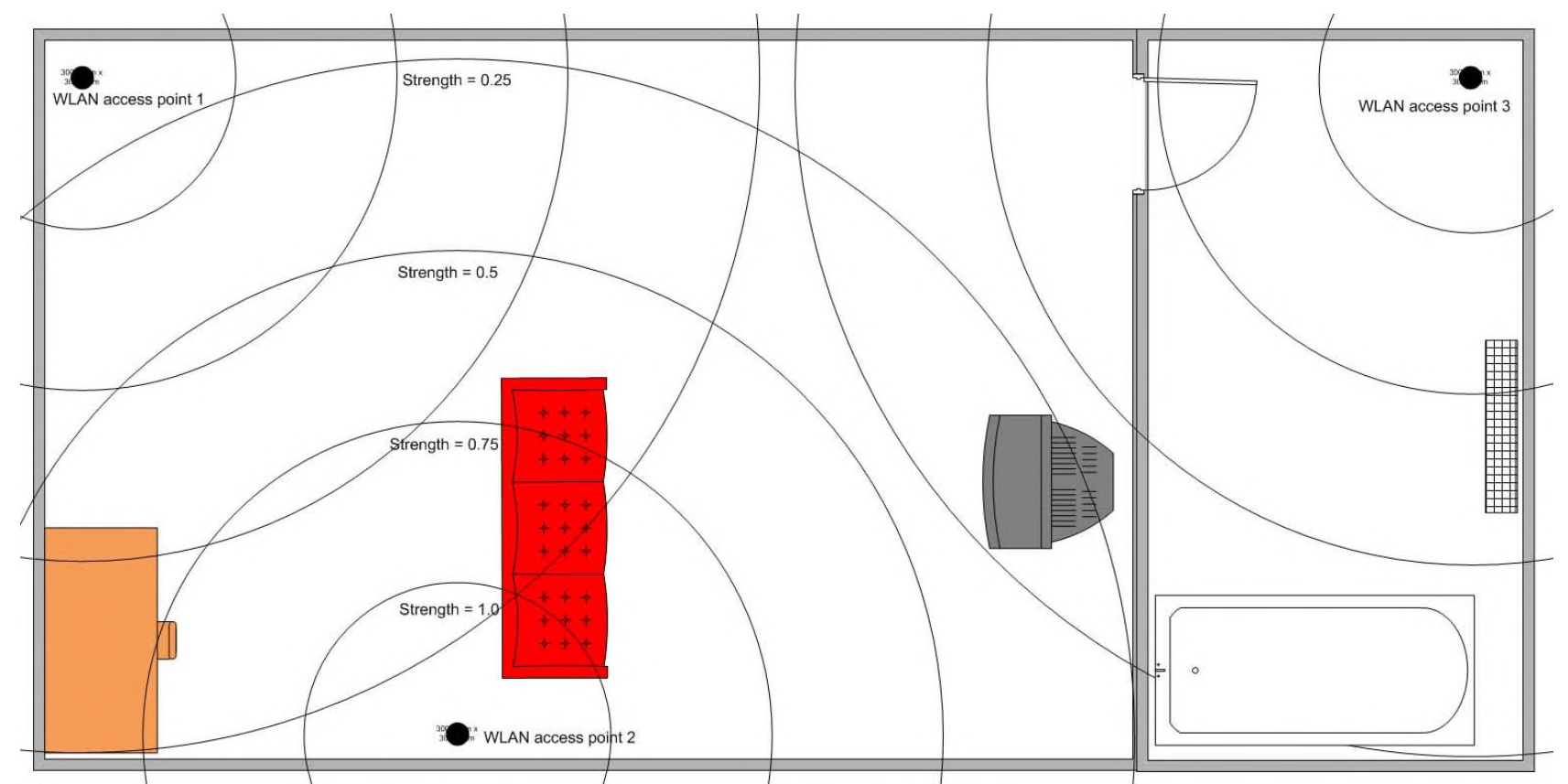

Figure 2. Degression Model for Signal Strength of WLAN Access Points. Example for Access Point 2.

get a line of sight to the physical object, the user had to move about one meter. To cover most casual activities, users provided information afterwards about their behaviour and preferences when lying or standing.

The results of the reference user study led to the following three findings. (1) Users tend to switch to a specific mobile interaction technique dependent on location, activity and motivation. (2) The current location of the user is the most important criterion for the selection of a mobile interaction technique. (3) The user's motivation to make any physical effort is generally low.

\section{The Implementation of the Test Setting using the Hybrid \\ Simulation}

The reference study constitutes our benchmark for performing a similar test, this time making use of a hybrid simulation. Thus, we first modelled the living room and the bathroom and the required physical objects. Figure 4 shows the perspective of the avatar when sitting on the couch. In front of the avatar is the DVD player within line of sight. To the left of the avatar is the radio within touching distance. The idea is to select the different physical objects by using one of the three mobile interaction techniques. Once the user has selected one of the physical objects, the respective services are displayed and the user can select one of them by using the mobile phone's graphical user interface. In the following we shortly describe the implementation of the three different mobile interaction techniques.

\section{Implementation of Scanning}

We applied MoPeDT to generate the pervasive interface for the mobile phone that supports scanning. It is completely realised on the real mobile phone (see Figure 3 ) and therefore no adaptation in the virtual world is required. The user navigates through different screens and finally selects the intended physical object in order to use a service for this object. Thus, scanning is quite similar to our reference study.
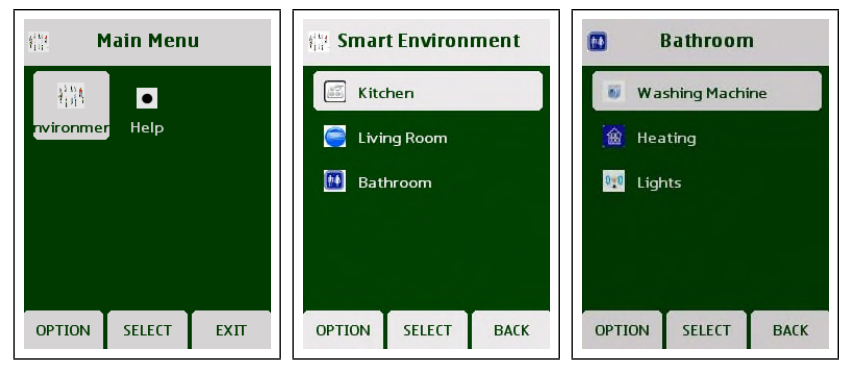

Figure 3. Screens to perform scanning.

\section{Implementation of Pointing}

In contrast to scanning, pointing requires a direct user interaction with the physical objects. Figure 4 shows the implementation of pointing in a hybrid simulation. The user applies the virtual mobile phone to point at a physical object in order to perform the selection. The virtual phone can be positioned by pressing the four navigation keys. By hitting the 'PgUp' key, an IR beam is emitted that is registered by the IR sensor of the virtual physical object. The information about the object's ID is then transmitted to the server of MoPeDT's architecture that forwards this context to the real phone and the application running on it. Now, the mobile phone loads the services of the selected object and displays them on the real phone. 


\section{Implementation of Touching}

Touching is realised by getting very close to the physical object in the virtual world and touch it with the virtual mobile phone (see Figure 4). Once the user has touched the physical object, a script sends the identifier to MoPeDT's server and the mobile phone as described for pointing.
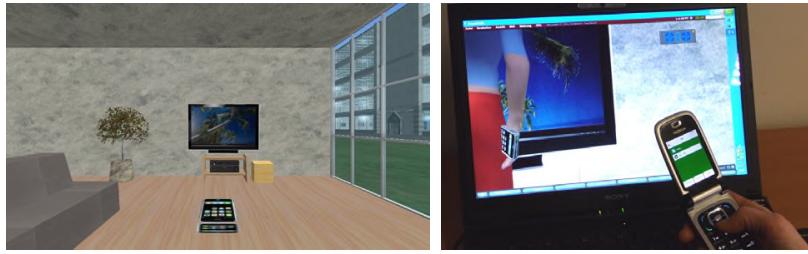

Figure 4. Pointing (left) and Touching (right)

\section{USER STUDY WITH A HYBRID SIMULATION}

After having implemented a hybrid simulation based on the idea of the reference study, we conducted a user study. First, we describe the experimental setting, then we report the user study and finally we illustrate our results.

\section{Experimental Setting}

The main objective of the experiment was finding out whether hybrid simulations of pervasive environments via Second Life can potentially be used as an evaluation method in the development process of a pervasive interface for mobile phones. In this context, benefits and problems should also be revealed by gaining practical experience. To address these aspects, we used our reference study and implemented a test setting. Based on this test bed, we deployed the experimental setting of the reference study, conducted a user experiment and compared the results.

\section{Hypotheses:}

We used the findings from our reference user study and formulated them as the following hypotheses, falling into three categories.

- H-1: Similar to the reference study, the users also tend to switch their mobile interaction technique based on their contextual situations when evaluating them with a hybrid simulation.

- H-2: Similar to the reference study, location is also the most important contextual criteria for selecting a mobile interaction technique when evaluating them with a hybrid simulation.

- H-3: Similar to the reference study, the user's motivation to make any physical effort is also generally low when evaluating them with a hybrid simulation.

\section{Independent and Dependent Variables}

In order to investigate our hypotheses, we defined independent and dependent variables. The independent variables are location and activity with the different levels of our reference study. Thus, the participants of our user study had to perform exactly the same tasks as for the reference study. As dependent variables, we analysed the user's preference for a mobile interaction technique in the different settings of independent variables. Thus, the experimental design is an exact replication of the reference study.

\section{Executing the Experiment}

In this section we shortly describe how the user test was conducted.

\section{Pre-Experiment}

Before we started the experiment, each participant of our user study was introduced to the correct usage of the mobile phone and the three interaction techniques. Moreover, we introduced the Second Life simulation. Prior to the test, the subjects could freely exercise each interaction technique and the use of the Second Life environment.

\section{Experiment}

We conducted the experiment with 20 subjects aged 23 to 32 with an average age of 27.25. The Second Life environment ran on an ordinary computer that required no special hardware capabilities. The participants of our study could navigate through the virtual world using the avatar to trigger the different contexts of pointing and touching. The pervasive interface ran on a Nokia 6131 NFC that could be used to perform scanning and to retrieve the services for incoming contexts triggered via touching or pointing. After the explanation of the mobile interaction techniques and the virtual test setting, we sat the avatar on the couch in Second Life. This was always the starting position for each task. Now, the participants of our study had to complete the four tasks described earlier. After each task, we asked them about their attitude if the avatar would stay beside the couch or lie on the couch instead of sitting. Therefore, the subjects had to fill out a questionnaire that addressed the different test settings. This questionnaire was identical to the reference study.

\section{Results}

For the hybrid simulation and the reference study, location could be identified as the crucial contextual factor for the decision of an interaction technique. An ANOVA test revealed these differences in location to be highly significant for the reference study (touching: $\mathrm{F}=19.225$, $p<0.01$, pointing: $\mathrm{F}=123.36, p<0.01$, scanning: $\mathrm{F}=10.769, p<0.01)$. A similar result was obtained with the hybrid simulation. Again, location was the crucial contextual factor that dominated the choice of an interaction technique with significant differences depending on the location (touching: $\mathrm{F}=12.013, p<0.01$, pointing: $\mathrm{F}=39.2, p<0.01$, scanning: $\mathrm{F}=9.604, p<0.01)$. No effect was found for the activity, i.e. it did not matter if the user was sitting, standing or lying. A post-hoc test revealed the dependencies between locations and 
interaction techniques. Touching was preferred in scenario 2, where the user is close to the desired object. Pointing is preferred in scenario 1 , where the user is around 3 meters from the object but the object is in her line of sight. Scanning at last is clearly preferred if the object is in another room. There is a tendency to also prefer scanning if the object is in the same room but not in the line of sight (scenario 4), but this preference is not significant. Scenario 4 reveals that the chosen technique might be dependent on the activity but the results are not conclusive in either study.

The findings from our hybrid simulation are comparable to our reference study. (H-1) They provided evidence for our first hypothesis: Similar to the reference study, the users also tend to switch their mobile interaction technique based on their contextual situations when evaluating them with a hybrid simulation. None of the participants used the same interaction technique in each tasks. Each of the participants assessed the situation and balanced reasons which interaction technique would fit best to which context. (H-2) We also could corroborate the second hypotheses: Similar to the reference study, location is also the most important contextual criteria for selecting a mobile interaction technique when evaluating them with a hybrid simulation. The execution of the user test with the hybrid simulation led to the result that location is the most important context factor which influences the decision for an interaction technique. In all four tasks the users tended to use the interaction technique dependent on the location of the avatar and the physical objects. If touching or pointing were possible they preferred these techniques. If there was no line of sight, the subjects tended to switch to scanning in order to prevent movements of the avatar. (H-3) The third hypotheses could also be partly proved: Similar to the reference study, the user's motivation to make any physical effort is also generally low when evaluating them with a hybrid simulation. The subject's motivation to spend physical effort was almost as low as in the reference study. But, in the hybrid simulation more subjects were willing to move the avatar in Second Life for performing touching or pointing, however, this difference is not statistically significant. A higher subject's motivation to spend physical effort is not completely surprising when using the hybrid simulation because the subjects did not have to actually move themselves but just navigate their avatar through the environment which is not comparable in effort to the real setting.

\section{DISCUSSION OF THE HYBRID SIMULATION}

Our user study showed that we gained very similar knowledge about the user's behaviour from results of the hybrid simulation compared to results from the reference study. Consequently, a first indicator points to the assumption that the hybrid simulation seems to be an appropriate evaluation method for early stages of the design process. Detailed benefits and problems of the method, however, must also be addressed in order to get a deeper insight whether the hybrid simulation is really meaningful. The now described advantages and disadvantages mainly base on our gained practical experience by the execution of a user study as a hybrid simulation.

In some points the hybrid simulation benefits compared to a traditional laboratory setting and virtual simulation alone approach. (1) Compared to a traditionally laboratory setting there is no need to physically rebuild the user's environment in a laboratory anymore. Thus, the designers can save money and time. (2) Relying on the hybrid simulation, even initial ideas of pervasive interfaces for mobile phones can easily and efficiently be mediated and investigated because the mobile application can be tried out and demonstrated in the corresponding simulated pervasive computing environment. (3) Another benefit is the ease of changing the environment. Different models of physical objects can rapidly be generated, modified and deleted. Thus, different settings of a pervasive environment can be arranged and tested in user studies. Using Second Life as virtual world adds further advantages. (4) Due to its widespread use, it is known to a great number of users who do not have to be introduced to the specifics of using the virtual environment. (5) A further advantage is the mobility of the test setting. Because the application realises a multi player platform over the internet, it can be accessed anywhere anytime. Thus, user tests can be run outside the laboratory in the user's familiar surroundings. (6) This can also reduce the organisational effort of subject recruiting since the subjects do not need to be physical present anymore that is similar to the execution of online surveys. Consequently, user studies can quick and easily be conducted with a large number of participants. Second Life has attracted a large number of users. These are potential test users for our interaction concepts. Of course some restrictions apply like the necessity of compatible mobile devices. (7) Finally, in contrast to virtual simulation alone approach, the hybrid simulation also arise the benefits that the evaluation can be performed more similar to the real setting. The users can directly interact with the real mobile phone which can increase the level of immersion.

Despite these promising benefits, there are also problems. Of course, there inevitably is an offset between a real world setting and a hybrid simulation. (1) The user moves virtually instead of physically which means a break because the user requires less motivation and less physical effort to move and explore the virtual setting. (2) A further problem of the hybrid simulation is the level of immersion for the mobile interaction. In our user study we applied scanning, pointing and touching as interaction techniques. Scanning is easy to evaluate with a hybrid simulation because it is completely realised on the mobile phone and therefore quite similar to the real usage but techniques, such as pointing and touching lead to a further break because they in- 
evitably require interactions with the pervasive environment. Our implementation of pointing is fully realised in the virtual world but instead should preferably be realised in the real world to reduce breaks to the real usage of the phone. We came up with the idea to replace our implementation of pointing and instead use the accelerometer of the mobile phone to point towards the screen of the virtual world simulation for selections of the objects, such as the DVD player. (3) Having too many interactions in the virtual simulation also leads to a problem of usability. Sometimes users had problems to perform pointing in our setting because it required the knowledge of key sequences. (4) A last problem is to generate the pervasive environment as realistic as the real setting. Developers require appropriate skills to virtually model the pervasive environment and set up the whole system to run a hybrid simulation.

\section{CONCLUSION}

In this paper, we investigated the hybrid simulation as an evaluation method in early stages development of pervasive interfaces for mobile phones. First, we described how to build this kind of simulation. Then as a first research step, we applied our prototypical setting of a smart environment and conducted a comparative user study in order to find potential problems and benefits of the method. Potentially, the hybrid simulation has many benefits, such as it can easily address a lot of subjects which can save time and money when organising and performing user evaluations. However, there are also problems which have to be considered, such as the level of immersion for the user. The experience we gained through the execution of a hybrid simulation points to a need to keep as many user interactions as possible in the real world and try to simulate as less user interactions in the virtual world as required. In that way, the offset between the two worlds can potentially be reduced and consequently the quality of the results improved. In future work we will address the mentioned problems more detailed to find appropriate solutions. But despite these problems, we already consider the hybrid simulation as a very promising method to improve the user-centred development of applications in the context of Pervasive Computing.

\section{ACKNOWLEDGEMENTS}

This research is partly sponsored by the research unit "OC-Trust" (FOR 1085) of the German Research Foundation (DFG).

\section{REFERENCES}

1. Rafael Ballagas, Jan Borchers, Michael Rohs, and Jennifer G. Sheridan. The smart phone: A ubiquitous input device. IEEE Pervasive Computing, 5:70-77, 2006.

2. John J. Barton and Vikram Vijayaraghavan. Ubiwise: A ubiquitous wireless infrastructure simulation environment, tech. report hpl2002-303. HP Labs, 2002, 2002.
3. Marco de Sá and Luís Carriço. Low-fi prototyping for mobile devices. In CHI '06: CHI '06 extended abstracts on Human factors in computing systems, pages 694-699. ACM, 2006.

4. Marco de Sá and Luís Carriço. Lessons from early stages design of mobile applications. In MobileHCI '08: Proceedings of the 10th international conference on Human computer interaction with mobile devices and services, pages 127-136. ACM, 2008.

5. Anind K. Dey. Understanding and using context. Personal Ubiquitous Comput., 5(1):4-7, 2001.

6. Henry B. Duh, Gerald C. B. Tan, and Vivian H. Chen. Usability evaluation for mobile device: A comparison of laboratory and field tests. In MobileHCI '06: Proceedings of the 8th Conference on Human-Computer Interaction with Mobile Devices and Services, pages 181-186. ACM Press, 2006.

7. Gita Gopal, Tim Kindberg, Tim Kindberg, and et al. John Barton. People, places, things: web presence for the real world. In In proceedings WMCSA2000., pages 365-376, 2000.

8. Lada Gorlenko and Roland Merrick. No wires attached: Usability challenges in the connected mobile world. IBM Syst. J., 42(4):639-651, 2003.

9. Mieke Haesen, Joan De Boeck, Karin Coninx, and Chris Raymaekers. An interactive coal mine museum visit: prototyping the user experience. In HSI'09: Proceedings of the 2nd conference on Human System Interactions, pages 543-550, Piscataway, NJ, USA, 2009. IEEE Press.

10. Jonna Häkkilä and Minna Isomursu. User experiences on location-aware mobile services. In OZCHI '05: Proceedings of the 17th Australia conference on Computer-Human Interaction, pages 1-4. Computer-Human Interaction Special Interest Group (CHISIG) of Australia, 2005.

11. Jonna Häkkilä and Jani Mäntyjärvi. Developing design guidelines for context-aware mobile applications. In Mobility '06: Proceedings of the 3rd international conference on Mobile technology, applications \& systems, page 24. ACM, 2006.

12. Morten Hertzum. User testing in industry: A case study of laboratory, workshop and field tests. In In A. Kobsa and C. Stephanidis (Eds.), User interfaces for all. Proceedings. 5. ERCIM workshop, pages 59-72, 1999.

13. Stephanie Houde and Charles Hill. What do prototypes prototype? Handbook of Human-Computer Interaction.

14. Dongsik Jo, Ungyeon Yang, and Wookho Son. Design evaluation using virtual reality based 
prototypes: towards realistic visualization and operations. In MobileHCI '0\%: Proceedings of the 9th international conference on Human computer interaction with mobile devices and services, pages 246-258. ACM, 2007.

15. Anne Kaikkonen, Aki Kekäläinen, Mihael Cankar, Titti Kallio, and Anu Kankainen. Usability testing of mobile applications. Journal of Usability Studies, 1(1):4-17, November 2005.

16. Eeva Kangas and Timo Kinnunen. Applying user-centered design to mobile application development. Commun. ACM, 48(7):55-59, 2005.

17. Jesper Kjeldskov and Connor Graham. A review of mobile hci research methods. In Mobile $H C I$, pages 317-335, 2003.

18. Jesper Kjeldskov, Connor Graham, Sonja Pedell, Frank Vetere, Steve Howard, Rine Balbo, and Jessica Davies. Evaluating the usability of a mobile guide: The influence of location, participants and resources. Behaviour and Information Technology, 24:51-65, 2005.

19. Jesper Kjeldskov and Jan Stage. New techniques for usability evaluation of mobile systems. International Journal of Human-Computer Studies, 60(5-6):599-620, May 2004.

20. Sangyoon Lee, Tian Chen, Jongseo Kim, Sungho Han, Zhi-geng Pan, and Gerard J. Kim. Affective property evaluation of virtual product designs. In VR '04: Proceedings of the IEEE Virtual Reality 2004, page 207. IEEE Computer Society, 2004.

21. Karin Leichtenstern and Elisabeth André. The assisted user-centred generation and evaluation of pervasive interfaces. In 3rd European Conference on Ambient Intelligence (AmI), 2009.

22. Karin Leichtenstern and Elisabeth André. Mopedt: features and evaluation of a user-centred prototyping tool. In EICS '10: Proceedings of the 2nd ACM SIGCHI symposium on Engineering interactive computing systems, pages 93-102. ACM, 2010.

23. Joshua Lifton and Joseph A. Paradiso. Dual Reality: Merging the Real and Virtual. In Proceedings of the First International ICST Conference on Facets of Virtual Environments (FaVE), July 2009.

24. Tony Manninen. Multimedia game engine as distributed conceptualisation and prototyping tool contextual virtual prototyping. In Proceedings IMSA2000 Conference, Las Vegas, NV, IASTED/ACTA Press 2000; 99104, pages 7-8. University Press.

25. Ji-Ye Mao, Karel Vredenburg, Paul W. Smith, and Tom Carey. The state of user-centered design practice. Commun. ACM, 48(3):105-109, 2005.
26. Ricardo Morla and Nigel Davies. Evaluating a location-based application: A hybrid test and simulation environment. IEEE Pervasive Computing, 3(3):48-56, 2004.

27. Brad Myers, Scott E. Hudson, and Randy Pausch. Past, present, and future of user interface software tools. ACM Trans. Comput.-Hum. Interact., $7(1): 3-28,2000$.

28. Jakob Nielsen and Thomas K. Landauer. A mathematical model of the finding of usability problems. In CHI '93: Proceedings of the SIGCHI conference on Human factors in computing systems, pages 206-213. ACM Press, 1993.

29. Antti Oulasvirta. Finding meaningful uses for context-aware technologies: the humanistic research strategy. In $C H I$ '04: Proceedings of the SIGCHI conference on Human factors in computing systems, pages 247-254. ACM Press, 2004 .

30. Antti Oulasvirta, Esko Kurvinen, and Tomi Kankainen. Understanding contexts by being there: case studies in bodystorming. Personal Ubiquitous Comput., 7(2):125-134, 2003.

31. Christian Pallay, Matthias Rehm, and Ekaterina Kurdyukova. Getting acquainted in second life: human agent interactions in virtual environments. In $A C E$ '09: Proceedings of the International Conference on Advances in Computer Enterntainment Technology, pages 36-43. ACM, 2009.

32. Vinny Reynolds, Vinny Cahill, and Aline Senart. Requirements for an ubiquitous computing simulation and emulation environment. In InterSense '06: Proceedings of the first international conference on Integrated internet ad hoc and sensor networks, page 1. ACM, 2006.

33. Stephanie Rosenbaum, Janice Anne Rohn, and Judee Humburg. A toolkit for strategic usability: results from workshops, panels, and surveys. In CHI '00: Proceedings of the SIGCHI conference on Human factors in computing systems, pages 337-344. ACM, 2000.

34. Enrico Rukzio, Karin Leichtenstern, Vic Callaghan, Paul Holleis, Albrecht Schmidt, and Jeannette Chin. An experimental comparison of physical mobile interaction techniques: Touching, pointing and scanning. pages 87-104. 2006.

35. Ichiro Satoh. Flying emulator: Rapid building and testing of networked applications for mobile computers. In in Proceedings of Conference on Mobile Agents (MA2001), LNCS, pages 103-118. Springer, 2001.

36. Mark Weiser. The computer for the 21st century. Scientific American, February 1991. 